\title{
3D printed flexible substrate with pneumatic driven electrodes for health monitoring
}

\author{
Martin Schubert, Sabine Friedrich, Karlheinz Bock \\ Electronics Packaging Laboratory \\ Technische Universität Dresden \\ Dresden, Germany \\ martin_schubert@tu-dresden.de
}

\author{
Daniel Wedekind, Sebastian Zaunseder, \\ Hagen Malberg \\ Institute of Biomedical Engineering \\ Technische Universität Dresden \\ Dresden, Germany
}

\begin{abstract}
Telemedical methods enable remote patient monitoring and healthcare at a distance. Besides, fitness tracker and sport watches are currently trending electronic products to generate awareness of health parameters in daily life. Especially, the long-term and continuous measurement of electrophysiological signals such as electrocardiogramm (ECG) becomes increasingly attractive for telemedical applications. Typically used disposable $\mathrm{Ag} / \mathrm{AgCl}$ wet electrodes for good skinelectrode contact can potentially cause skin irritation and rashes. This paper presents a low cost, individual and flexible substrate for skin electrodes to be applied in future consumer electronic or professional applications. It enables an alternative contact method of the electrode to the skin by applying a pressure during the measurement and hence good contact. If no measurement is needed pressure can be released and the electrode loses skin contact. The 3D printed polymer module is $4 \mathrm{~mm}$ thick and comprises a pressure chamber, silver electrodes and insulation layer. The airtight printed membrane of flexible filament, which expands when inflating the chamber, may be printed in different thicknesses and shapes, much thinner than the present $4 \mathrm{~mm}$. This enables a high individuality for various applications. Pressure up to $150 \mathrm{kPa}$ was applied and leads to dilatation of $1400 \mu \mathrm{m}$. First tests on skin when measuring electrodermal activity (EDA) show promising results for future applications.
\end{abstract}

Keywords-3D printed, flexible substrate, pneumatic electrode, low cost, skin contact

\section{INTRODUCTION}

Telemedical methods are aspiring to deliver human support in many situations for the internet of things and for industry 4.0. In-particular they enable healthcare at a distance, remote patient monitoring with easier inter-medical communication to improve the quality of patient's treatment and higher access to healthcare, particularly in areas with high patient-to-doctor ratio [4]. Telemedical methods become advantageous for the collection of clinical trial data and especially within treatment intervals, when patients left the healthcare environment but still can be remotely monitored on various health parameters for emergencies, pre- or aftertreatment [3]. Also health care expenses can be lowered if parameters like respiratory rate, electrocardiographic data (ECG) or electrodermal activity (EDA) can be gained remotely without consulting a doctor [6].
For this reason the market is developing from rigid, stationary systems towards more adaptable, skin-mounted and wearable electronics [7]. Skin electrodes should be lightweight, not bothering, easy to handle, biocompatible, flexible and capable of delivering good signal quality $[1,11]$. Such paradigm change becomes visible on current trending fitness tracker and sport watches, which help to generate certain awareness for health parameters in public. Those devices, mostly for consumer market, need to be low cost on the one hand but reliable and functional on the other. Those contrary demands lead already in exceptional cases to the application of not fully suitable materials for continuous skin contact and resulted in allergic reactions of users [8]. Also in professional environment long term electrode-skin contact may cause issues. Typically skin electrodes can be roughly classified in wet and dry electrodes [1]. Most often used disposable $\mathrm{Ag} / \mathrm{AgCl}$ wet electrodes potentially lead to skin irritation and rashes due to the long-term application of the required gel for better electrical contact and the adhesive of the bandages may trigger irritations [1]. Therefore metal or carbon electrodes on flexible and stretchable substrates were developed and show promising results towards long-term stability, biocompatibility and signal quality [2]. Metal electrodes in combination with occurring sweat may also lead to incompatibilities due to galvanic processes that generate unexpected ions [9]. Additionally, impurities in materials or alloys for example stainless steel electrodes may trigger allergic reactions like for example nickel allergy [10]. Nevertheless, tight attachment of skin electrodes and good electrode-skin contact is essential for the functionality of the electrodes during measurement to ensure a high signal-to-noise ratio and to avoid artefacts.

This paper's approach is an alternative, on demand electrodeskin contact for measuring biosignals in future healthcare applications. In detail, the expandable electrode module (Fig. 1) comprises two equal pressure chambers with silver electrodes and insulation layer and was primarily designed for evaluation of the manufacturing process. This first set-up works with two basic syringe pumps which deliver the pressure to inflate the chambers. The module can be equipped with valves and micropumps replacing the syringes in order to perform in a wearable system. 

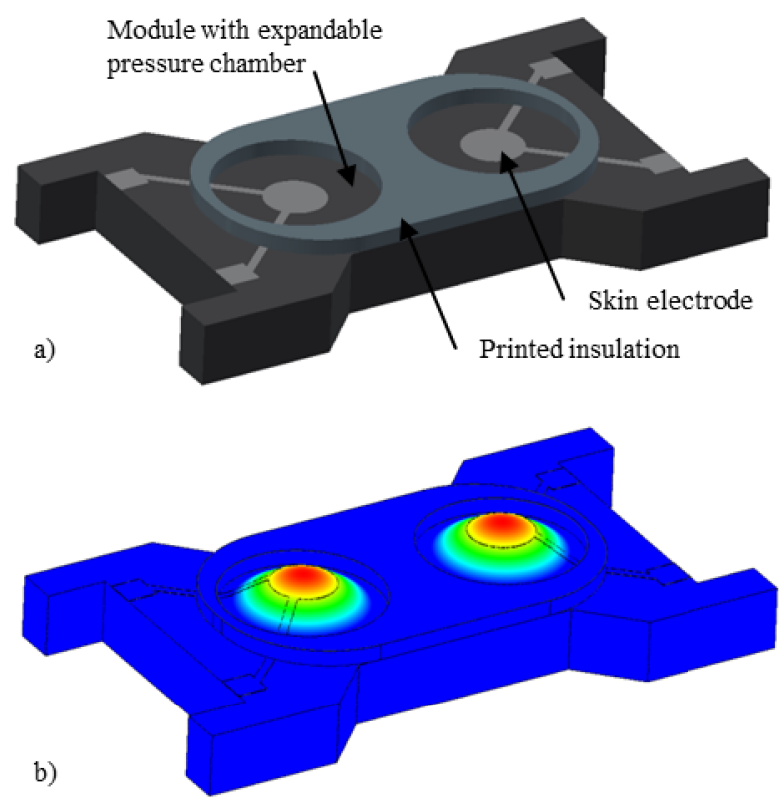

Fig. 1. Schematic drawing of the expandable pressure module with a) undilated $b$ ) dilated membrane

In case of measurement, the membrane on top of the chamber dilates by applying a positive pressure to elastically deform the chamber shape and the electrodes are pressed onto the skin where an electrical contact is established. Within the measurement intervals, pressure is released and the electrode pulls back elastically and opens the contact. Depending on the elastic properties of compound material used to print this electrode chamber, the chamber can be even actively evacuated as it is also the case in this first set-up. The dilatation of the membrane can be varied depending on the applied pressure that can be used to ensure a good contact even in e.g. moving measurement conditions by pressing the electrodes tighter onto the skin. The module was fully printed and therefore comprises all advantages of additive manufacturing such as low cost, quickly up scalable and individuality for various application such as fully printed pneumatic valves or microfluidic applications.

\section{ADDITIVE INTEGRATION}

The expendable electrode module was $3 \mathrm{D}$ printed using the fused deposition modelling (FDM) printer Fullscale XT Plus

a) 3D Printing of substrate and pressure chamber

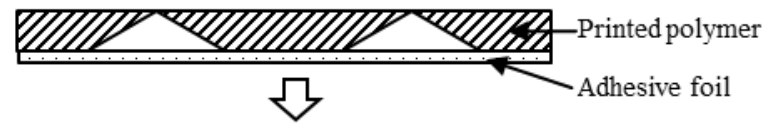

b) Screen printing of silver paste

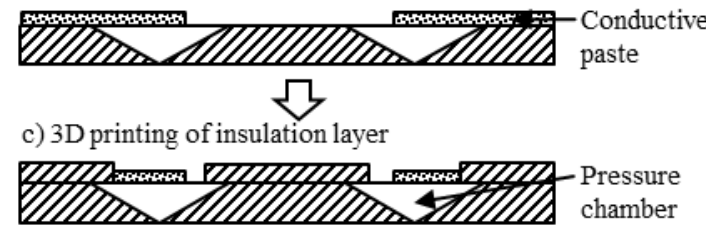

Fig. 2. Process steps for printing of the module

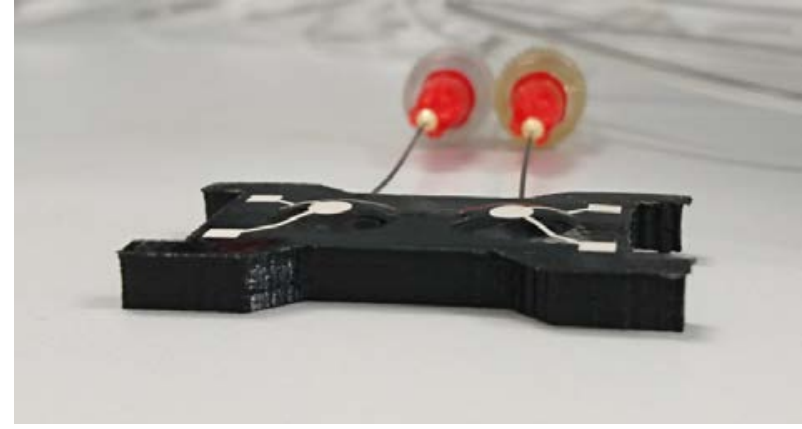

Fig. 3. Testing the dilatation of the module after fabrication (compare with Fig. 2 b)

from Mankati, working with filaments of $2.85 \mathrm{~mm}$ diameter. The flexible filament is PolyFlex ${ }^{\mathrm{TM}}$ from Polymakr LLC. It's thermoplastic polyurethane (TPU) with following properties [5]:

- $\quad$ Shore hardness 95A

- $\quad$ Tensile strength $(29 \pm 2.8) \mathrm{MPa}$

- $\quad$ Elongation at break $(330.1 \pm 14.9) \%$.

The manufacturing process of the module is shown in Fig. 2. First the filament was printed at $230{ }^{\circ} \mathrm{C}$, a speed of $45 \mathrm{~mm} / \mathrm{s}$ and a layer height of $100 \mu \mathrm{m}$. Within this step the basic electrode module with pressure chamber and membrane was printed. The module is $4 \mathrm{~mm}$ high, $40 \mathrm{~mm}$ long and $22 \mathrm{~mm}$ wide. In this first printing step the setting of the distance between the nozzle and the build plate mainly determines the membrane thickness and hence the dilatation rate of the printed module. An arithmetic average surface roughness of about $\mathrm{R}_{\mathrm{a}}=1.5 \mu \mathrm{m}$ was achieved by printing on an adhesive foil. In the next step, the electrodes were screen printed using polymeric silver paste C5029 from DuPont de Nemours $\mathrm{GmbH}$ and a MPM SPT screen printer. The paste was cured $60 \mathrm{~min}$ at $60{ }^{\circ} \mathrm{C}$. The electrode area is $12.6 \mathrm{~mm}^{2}$ and two contact pads were connected to the electrode for redundant contact. The layer height is in average $23.1 \mu \mathrm{m}$. The last step was again FDM printing of the insulation layer of a thickness of $(0.7$ to 0.9$) \mu \mathrm{m}$ using PolyFlex ${ }^{\mathrm{TM}}$ on top of the electrodes. Here the nozzle distance is to be set not to damage the previously screen printed electrodes. For contacting, the electrodes' wires were connected to the pads using conductive adhesive H20E (Epoxy Technology Inc.).

\section{METHODS FOR CHARACTERIZATION}

The expandable electrode module was characterized regarding pressure depending dilatation of the membrane for potential skin electrode application. Therefore the pressure chambers were connected from outside through syringe needles to apply the air inside the module (Fig. 3). The flexible material automatically seals the puncture airtight. The pressure was generated with a syringe with $50 \mathrm{ml}$ volume. The main advantage of using syringes is to be able to apply a positive and a negative pressure through pushing or pulling at the syringe. A linear axis was used for repetitive force at the syringe. 


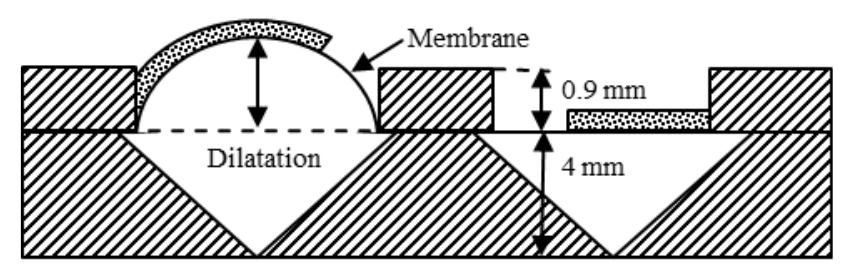

Fig. 4. Cross section of the module showing

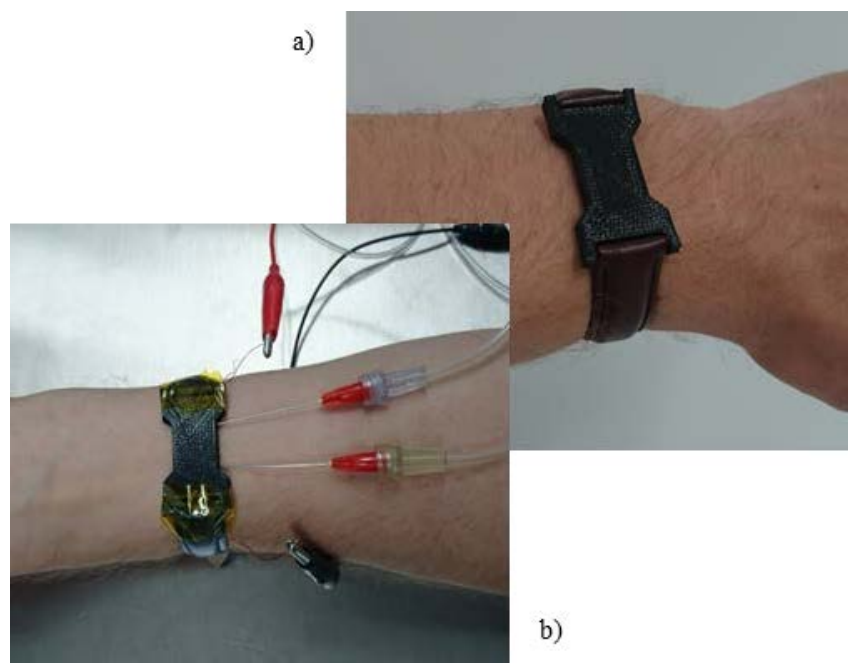

Fig. 5. Due to the open formfactor the module is easily connected to an wristband for future applications. a) As substrate for smartwatches or b) for EDA measurments at the bottom of the forearm

The pressure was controlled using a digital manometer DP200 from Mecotec and an analog one from Festo AG with graduation of 100 mbar. The dilatation as shown in Fig. 4 was determined with the $\mu$ scan profilometer (NanoFocus AG) using the chromatic sensor CLA. The air leakage of the system was average $1.9 \mathrm{kPa}$ per $60 \mathrm{~s}$. Afterwards the membranes were cut out and the achieved thickness was measured.

Furthermore, the electrical resistance of the printed electrodes was measured. Therefore a module with $220 \mu \mathrm{m}$ thick membrane was connected to the HM8118 LCR-Bridge (HAMEG Instruments). A standard copper-cladded FR4 substrate was pressed on top of the electrodes with a weight force of $0.9 \mathrm{~N}$. Any less weight was lifted by the force generated by the electrodes. Applying pressure of $75 \mathrm{kPa}$ to both pressure chambers establishes a connection between the electrodes and the copper. The resistance was measured over 40 press and release inflation cycles with a periodic time of $2 \mathrm{~s}$. Furthermore the resistance was evaluated over a period of $75 \mathrm{~s}$ while pressure was hold.

Regarding future applications (Fig. 5) the electrodermal activity was measured. A module with a $220 \mu \mathrm{m}$ thick membrane was connected to a wristband and placed at bottom side of the left forearm. The EDA measurement was performed with the GSR Amp (AD Instruments). It uses low, constantvoltage AC excitation (22 mV rms @ $75 \mathrm{~Hz})$ and MLT116F stainless steel finger electrodes $\left(480 \mathrm{~mm}^{2}\right)$. The standard electrodes were used as reference and also placed at the same location as the electrode module.

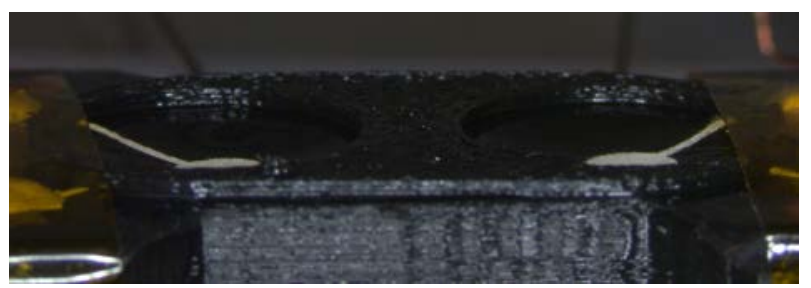

Fig. 6. View on the electrodes with applied underpressure of $-10 \mathrm{kPa}$

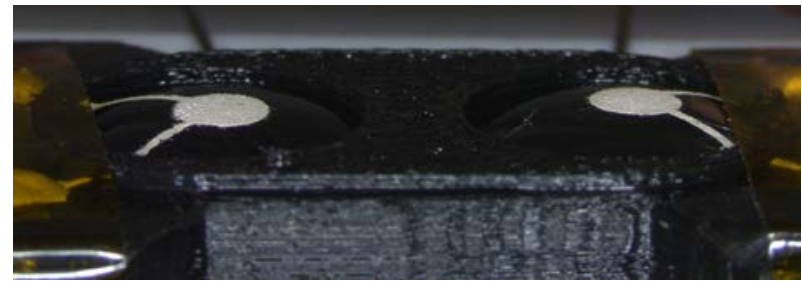

Fig. 7. View on the electrodes with applied overpressure of $75 \mathrm{kPa}$

\section{RESULTS}

The expandable pressure module was characterized for its properties for future skin electrode application. Fig. 6 and Fig. 7 show the printed expandable pressure module with isolation layer. In Fig. 6 an underpressure of $-10 \mathrm{kPa}$ was applied and leads to indrawn electrodes with maximum distance between skin and electrodes. In Fig. 7 an overpressure of $75 \mathrm{kPa}$ within the pressure chambers leads to a dilatation of the membrane and hence an electrode-skin contact in case of measurement. The shown gap between module and insulation layer represents difficulties of alignment. Fig. 8 shows the dilatation of a selection of different membranes in thickness depending on the applied pressure. The pressure was increased from ( 0 to $150) \mathrm{kPa}$ and then decreased to $-10 \mathrm{kPa}$. Within this pressure range only minor viscoelastic behavior occurred. This is noticeable in the hysteresis of the dilatation curve in Fig. 8, meaning lower dilatation shown by the increasing pressure (lower) curve compared to the decreasing pressure (higher) curve.

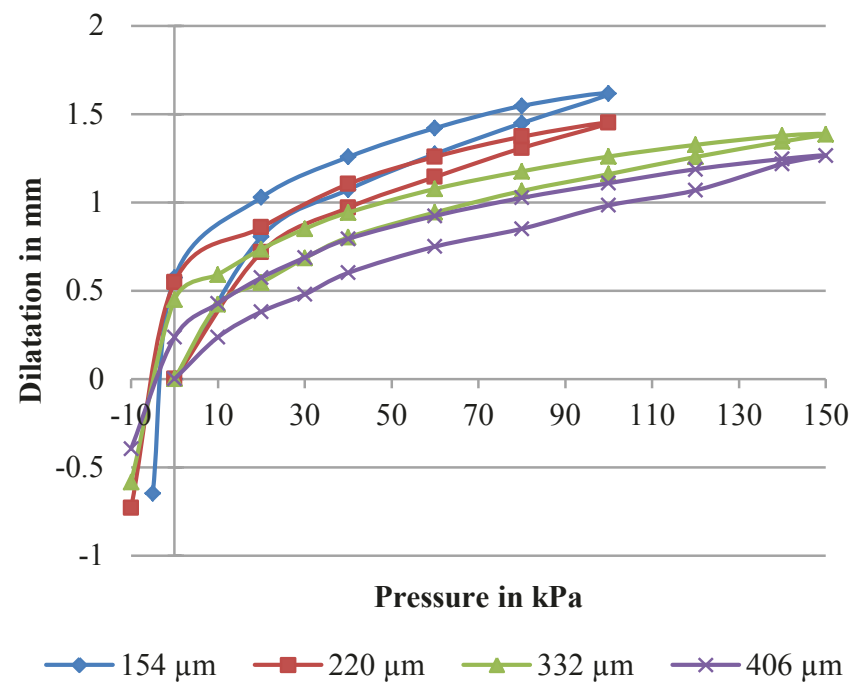

Fig. 8. Membrane dilatation of various membrane thicknesses 
Because of membranes with lower thickness showed irreversible deformation over $100 \mathrm{kPa}$, the utilized peak pressure of those is lower. In addition, those membranes showed more dilatation at lower pressure but also higher dilatation in general. For example, a $154 \mu \mathrm{m}$ membrane showed a dilatation of $1.62 \mathrm{~mm}$ at $100 \mathrm{kPa}$. A higher hysteresis compared to thicker membranes was not found. As expected the thickest membrane $(406 \mu \mathrm{m})$ showed the lowest maximum dilatation. For evaluating possible security issues through bursting of the pressure chambers a maximum pressure of $240 \mathrm{kPa}$ was applied. $332 \mu \mathrm{m}$ membranes reached plastic deformation but showed still partly reversible dilatation of $1.85 \mathrm{~mm}$. Membranes with lower thickness were irreversibly plastically deformed but did not burst.

Furthermore the electrical resistance between the screen printed electrodes was evaluated. Fig. 9 shows decreasing electrical resistance by roughly $9 \Omega$ over $75 \mathrm{~s}$ at a pressure of $75 \mathrm{kPa}$. After $45 \mathrm{~s}$ the final resistance of in average $16.0 \Omega$ was reached. The reason for decreasing resistance after inflation of the chambers is that the electrodes are connected quickly and with high pressure to the copper substrate. After some time due to elastic properties of the materials the electrode will cling to the copper and the resistance decreases. In Fig. 10 the resistance over 40 press and release inflation cycles is shown. The periodic time was $2 \mathrm{~s}$. As a result, the more cycles performed the higher the resistance of the electrode rises. As a consequence visible in the hysteresis of the electrodes, the membranes will not fully elastically contract back to their original shape. Hence, the silver paste will be stretched continuously more and more and its resistance rises.

For the modules first test as skin electrode the electrodermal activity was measured. Fig. 11 clearly shows the pressure dependency of the electrode-skin contact depicted as conductance over time because the skin conductance is proportional to the sweat secretion [12]. Three different pressures were applied. At a pressure of $-10 \mathrm{kPa}$ the electrodes do not reach electrical contact. At a pressure of $12 \mathrm{kPa}$ the electrical contact is established but still with low conductivity. At a pressure of $75 \mathrm{kPa}$ the whole electrode was fully pressed onto the skin with the result that the proband was able to feel the dilatation of the membrane. The highest conductivity was achieved with this pressure.

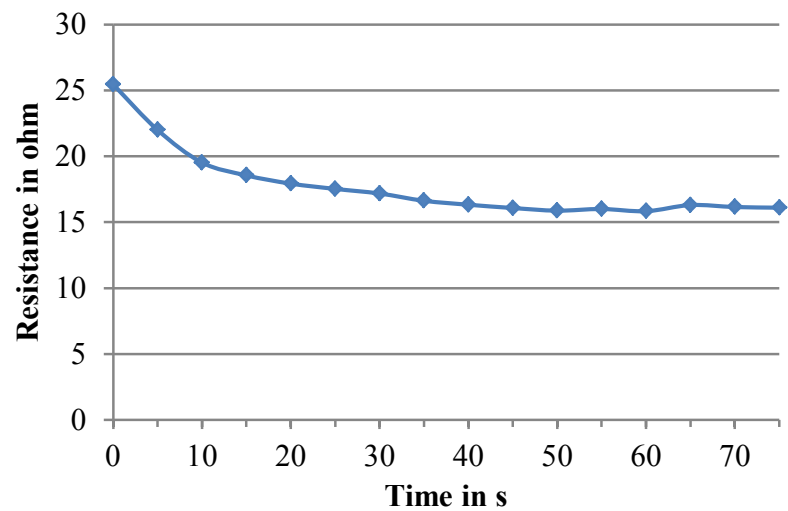

Fig. 9. Electrical resistance between the electrodes over $75 \mathrm{~s}$ hold contact to a copper substrate

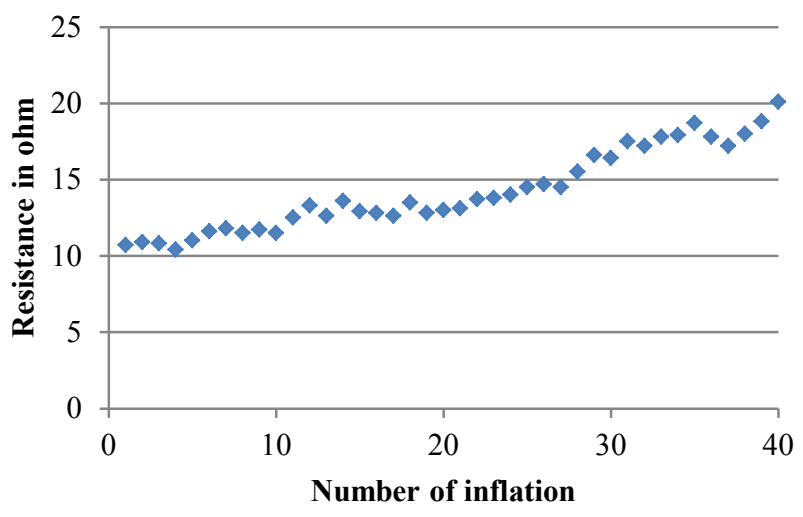

Fig. 10. Electrical resistance over 40 press and release inflation cycles with periodic time of $2 \mathrm{~s}$

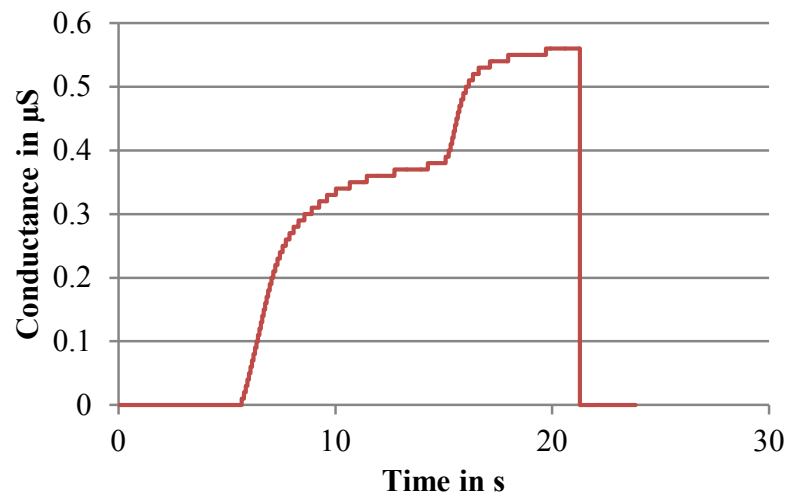

Fig. 11. Skin contact of the expandaple electrode module during EDA at different pressures

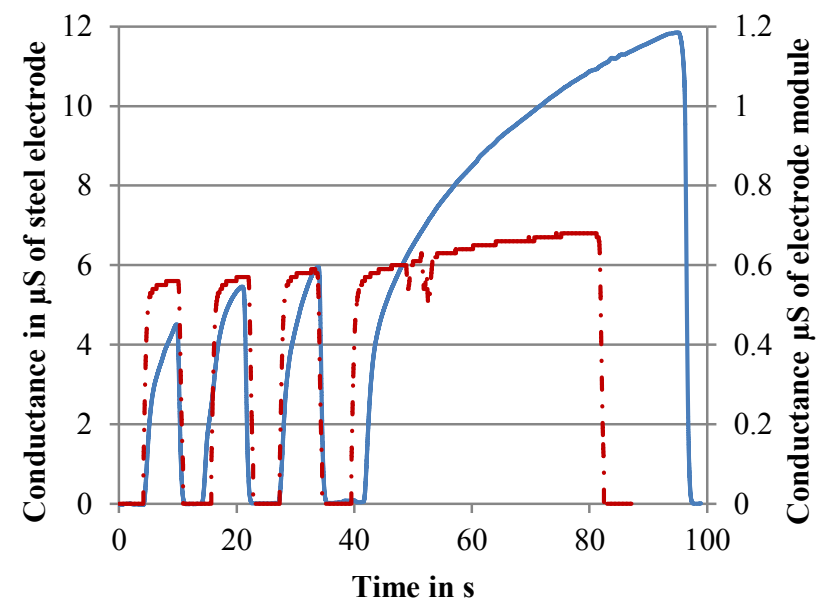

Steel electrode $\quad \cdots$ - Electrode module

Fig. 12. Comparison of steel electrode and electrode module with different signal amplitudes

Fig. 12 shows the EDA measurement using the standard steel electrode and the electrode module. To demonstrate the possible signal course during press and release cycles and hence contact and non-contact with both electrodes, the steel electrode has also been removed from the skin in a similar 
frequency. It can be seen that the electrode signal has similar shape to the standard electrodes only with a 10 times smaller amplitude. The major reason for this is assumed to be the electrode area. The steel electrodes are roughly 38 times larger compared to the printed silver electrodes. Furthermore, when measuring EDA relative changes are even more important than absolute changes [12]. During the EDA measurement also typical artifacts occurred, which can be seen in the diagram. The module was not yet able to waive moving artefacts through totally fixed position on the skin. However, an interesting observation is the temporal behavior of both electrodes. While the electrode module shows only slight variations after some seconds (approx. $10 \%$ change in the interval from $(5-45) \mathrm{s}$ after disposal), the steel electrode shows much stronger variations (about $100 \%$ in the same time interval). In case of intermittent artifacts the higher dynamicity of the electrode module might be of big advantage.

\section{CONCLUSION}

This paper presents an alternative, 3D printed, on demand, pressured contact electrode module for measuring biosignals for future healthcare applications. The module was characterized regarding its mechanical and electrical functionality and tested in a first on-skin application, the electrodermal activity measurement. It showed good properties for on-demand measurements with a good repeatability. Yet the hysteresis and reliability has to be further investigated. When the electrical contact was established the conductivity reaches a constant value after $45 \mathrm{~s}$ of contact. This should be considered for on-demand measurements. Furthermore the contact material for the electrodes needs improvement since the conductivity degraded with the number of inflations. Also long term measurements have to be performed to test for the elasticity of the pressure chamber and a pressure and control unit needs to be embedded within or mounted on the 3D printed device. The on demand electrodes module represents a low cost possibility with all advantages of 3D printing and therefore seems even suitable to be considered for disposable electrodes.

\section{ACKNOWLEDGMENT}

This work was supported by the European Social Fund (ESF) and the Free State of Saxony.

\section{REFERENCES}

[1] S. Yao and Y. Zhu; "Nanomaterial-Enabled Dry Electrodes for Electrophysiological Sensing: A Review," The Journal of The Minerals, Metals \& Materials Society, Volume 68, Issue 4, pp. 1145-1155, 2016

[2] W. Uter and H. Schwanitz, "Contact Dermatitis" 34, 230 (1996).

[3] Bundesärztekammer, „Ärztliche Priorisierung von Einsatzgebieten telemedizinischer Patientenversorgung," Entschließung and Positionspapier, 118. Deutscher Ärztetag, 2015

[4] D. Alajmi, S. Almansour and M.S. Househ, "Recommendations for implementing telemedicine in the developing world," in Informatics, Management and Technology in Healthcare, vol. 190, J. Mantas and A. Hasman, Eds. IOS Press, 2013

[5] Polymakr LLC, "PolyFlex ${ }^{\mathrm{TM}}$ Technical Datasheet," [Online] http://www.polymaker.com/wp-content/uploads/2015/06/PolyFlex_TDS -v1.pdf, visited on 01.07.17

[6] J. Lagasse, "Continuous monitoring tools could save hospitals $\$ 20,000$ per bed, report says," in HealthcareITNews, [Online] http://www.healthcareitnews.com/news/continuous-monitoring-toolscould-save-hospitals-20000-bed-report-says, visited 01.07.2017

[7] T. Vuorinen et al., "Printed, skin-mounted hybrid system for ECG measurements," 6th Electronic System-Integration Technology Conference (ESTC), 2016

[8] R. Abrams, "Fitbit says it will make changes to address complaints about allergic reactions," The New York Times, 17.10.2014, [Online] https://www.nytimes.com/2014/10/18/technology/personaltech/fitbitsays-it-will-make-changes-to-address-complaints-about-allergicreactions-.html, visited on 15.07.17

[9] L. A. Geddes and R. Roeder, "Criteria for selection of Materials for implanted electrodes," Annals of Biomedical Engineering, vol. 31, pp. 879-890, 2003

[10] J. P. Thyssen and T. Menne, "Metal Allergy-A review on exposures, penetration, genetics, prevalence, and clinical implications," Chemical Research in Toxicology, vol. 23, pp. 309-318, 2010

[11] M. Weder et al., "Embroidered electrode with silver/titanium Coating for Long-Term ECG Monitoring," Sensors, vol. 15, pp. 1750-1759, 2015

[12] Cornelia Setz et al., "Discriminating stress from cognitive load using a wearable EDA device," IEEE Transactions on information technology in biomedicine, vol. 14, 2010 